\title{
Decreased Response Contrast of Hadley Circulation to the Equatorially Asymmetric and Symmetric Tropical SST Structures during the Recent Hiatus
}

\author{
Juan Feng ${ }^{1}$, Jianping $\mathrm{Li}^{1,2}$, Yaqi Wang ${ }^{1}$, and Yipeng Guo ${ }^{3}$ \\ ${ }^{1}$ State Key Laboratory of Earth Surface Processes and Resource Ecology and College \\ of Global Change and Earth System Science, Beijing Normal University, Beijing, China \\ ${ }^{2}$ Laboratory for Regional Oceanography and Numerical Modeling, \\ Qingdao National Laboratory for Marine Science and Technology, Qingdao, China \\ ${ }^{3}$ School of Atmospheric Sciences, NanJing University, NanJing, China
}

\begin{abstract}
Variations of the Hadley circulation (HC) are influenced by the underlying sea surface temperature (SST). The contrasting response of the HC to meridional structure of SST is examined between two periods, one prior to and the other during the recent warming hiatus (i.e., 1979-1998 and 1999-2015). By decomposing the variations of $\mathrm{HC}$ and SST into equatorially asymmetric (HEA for HC; SEA for SST) and symmetric components (HES for HC; SES for SST), the HEA response to SEA and the HES response to SES are quantitatively analyzed. Multiple reanalyses consistently indicate that the ratio of the response of HEA to SEA with respect to that of HES to SES is clearly decreased in the period 1999-2015. This is because the response of HEA to SEA is suppressed in this period, while the response of HES to SES is enhanced, suggesting a corresponding change in the air-sea interactions involved during the two periods. Further examination found that the variation of SST over Pacific may play an important role in determining the reduced response ratio of $\mathrm{HC}$ to SST in the recent hiatus.

(Citation: Feng, J., J. Li, Y. Wang, and Y. Guo, 2017: Decreased response contrast of Hadley circulation to the equatorially asymmetric and symmetric tropical SST structures during the recent hiatus. SOLA, 13, 181-185, doi:10.2151/sola.2017-033.)
\end{abstract}

\section{Introduction}

Variations of the Hadley circulation (HC) are closely connected to the underlying thermal conditions (Feng et al. 2011; Sun and Zhou 2014). Previous studies have shown that the meridional gradient of sea surface temperature (SST) plays an important role in influencing the spatial structure of the HC. For example, theoretical models have shown that the strength and position of convergence in the lower troposphere is sensitive to the meridional structure of SST (e.g. Schneider and Lindzen 1977; Hou and Lindzen 1992), and numerical models suggested that the SST gradient is the primary factor determining the latitudinal structure of the HC in the planetary boundary layer (Numaguti 1994). Recently, using reanalysis data (i.e., the National Centers for Environmental Prediction/National Center for Atmospheric Research dataset (NCEP1)), Feng et al. (2013) pointed out that the structure of the meridional gradient of SST determines the spatial structure of the HC. Namely, the equatorially symmetric (asymmetric) SST anomalies are accompanied by equatorially symmetric (asymmetric) HC anomalies (Guo et al. 2016). In addition, Feng et al. (2016) has quantitatively assessed the changes in response amplitude of the $\mathrm{HC}$ to different SST meridional structures in the interannual

Corresponding author: Jianping Li, State Key Laboratory of Earth Surface Processes and Resource Ecology and College of Global Change and Earth System Science, Beijing Normal University, Beijing, 100875, China. E-mail: 1jp@bnu.edu.cn. (C2017, the Meteorological Society of Japan. scale. They found that the response amplitude of the $\mathrm{HC}$ to the equatorially asymmetric SST perturbations is about $\sim 5$ times larger than the response to equatorially symmetric SST perturbations during 1948-2013. Besides, reanalysis and model data have shown the widening of the HC from 1997 to 2012 is associated with a decreased meridional gradient of SST between the tropics and mid-latitudes (Adam et al. 2014). The above point further highlights the fundamental impacts of the meridional structure of SST on the HC.

However, global warming appears to have 'paused' in the past decade; that is the global surface temperature (including SST) shows a noticeable flat trend, or hiatus (Keenlyside et al. 2008). Several possible explanations have been suggested for this phenomenon. For instance, the contribution from sulfate aerosols (e.g. Booth et al. 2012), the North Atlantic Oscillation (Li et al. 2013), the eastern Pacific SST (Kosaka and Xie 2013), Pacific trade winds (England et al. 2014), the Interdecadal Pacific Oscillation (Dai et al. 2015), and the Pacific Decadal Oscillation (Trenberth and Fasullo 2013). Irrespective of the cause of the hiatus in the past decades, the changes in the temperature may alter the meridional distribution of SST (Adam et al. 2014), which may affect the HC in turn. Therefore, it is useful to examine the possible variations of SST meridional structure during the past decades and earlier, and to compare the responses of HC to different SST meridional structures between the periods prior to and during the hiatus.

In this study, to compare the responses of HC to SST meridional structure prior to and during the hiatus, the variations of $\mathrm{HC}$ and SST were linearly separated into the equatorially asymmetric and symmetric components following Feng et al. (2016) to examine their impacts during 1979-1998 and 1999-2005, respectively. The remainder of this paper is organized as follows. Section 2 describes the datasets and methodology. Section 3 demonstrates the contrasting responses of the HC to different SST meridional structures during the two periods. Section 4 contains a short discussion and our conclusions.

\section{Datasets and methodology}

\subsection{Datasets}

The global SST reanalysis datasets used were from the UK Met Office Hadley Centre sea ice and SST dataset gridded at $1^{\circ}$ $\times 1^{\circ}$ resolution (HadISST; Rayner et al. 2003), and the Extended Reconstructed SST version $3 \mathrm{~b}\left(\right.$ ERSST) on a $2^{\circ} \times 2^{\circ}$ grid (Smith et al. 2008). The atmospheric reanalyses were the NCEP1 (Kalnay et al. 1996) at $2.5^{\circ} \times 2.5^{\circ}$ resolution, the NCEP-Department of Energy Atmospheric Model Intercomparison Project reanalysis (NCEP2; Kanamitsu et al. 2002) at $2.5^{\circ} \times 2.5^{\circ}$ resolution, and the European Centre for Medium-Range Weather Forecasts (ECMWF) Re-Analysis Interim globally archived dataset (ERAI; Dee et al. $2011)$ at $0.75^{\circ} \times 0.75^{\circ}$ resolution. Since the global zonal mean is considered, the different resolutions of reanalyses are not interpolated onto a common grid. To compare the different responses 
of the HC to SST prior to and during the hiatus in the last decade, two periods (i.e., 1979-1998 and 1999-2015) are considered. The demarcation, 1998, corresponds exactly to a transition (i.e., turned strengthened linear trend into decreased trend) in the strength of the HC (Guo 2016) and temperature (England et al. 2014; Xing et al. 2016).

\subsection{Methodology}

The HC is characterized by the mass stream-function (MSF). To separately access the impacts of SST on HC, the spatial variations in SST and HC were linearly separated into two components following Feng et al. (2016); i.e., the equatorially symmetric (HES for HC, SES for SST) and asymmetric (HEA for HC, SEA for SST) components. The HES and HEA are defined as

$$
\begin{aligned}
& \operatorname{HES}(j)=\frac{\operatorname{MSF}(j)-\operatorname{MSF}(-j)}{2}, \\
& \operatorname{HEA}(j)=\frac{\operatorname{MSF}(j)+\operatorname{MSF}(-j)}{2} .
\end{aligned}
$$

The SES and SEA are defined as

$$
\begin{aligned}
& S E S(j)=\frac{S S T(j)+S S T(-j)}{2}, \\
& \operatorname{SEA}(j)=\frac{S S T(j)-S S T(-j)}{2},
\end{aligned}
$$

where $j$ and $-j$ correspond to the equatorially symmetric meridional locations of the grid points. The sum of the equatorially symmetric and asymmetric variations equals to its original variations. The definition of HES and HEA has opposite sign compared to those of SST because the MSF has the same and opposite sign across the equator for its asymmetric and symmetric components, respectively (Feng et al. 2017). Based on the decomposition shown, we obtain the interannual variations of the HEA, HES, SEA, and SES. Note that the dimensions of HEA/HES and SEA/ SES are same as their original variables of HC and SST.

Empirical orthogonal function (EOF) analysis was used to extract the principal mode of the variations of HEA, HES, SEA and SES after removing the annual cycle. The relationship between the HC and SST was investigated using correlation analysis. The regression was calculated using least squares linear regression. The statistical significance of the correlation and regression values was evaluated by means of a two-sided Student's $t$-test.

\section{Results}

Figure 1 shows the principal EOF mode (EOF1) of HEA during the two periods based on different reanalyses. An equatorially asymmetric mode dominates the variations of HEA in both periods, with ascending (descending) branch in the $\mathrm{SH}(\mathrm{NH})$ around $13^{\circ} \mathrm{S}\left(13^{\circ} \mathrm{N}\right)$. Although the intensity of this mode shows some differences across the reanalyses, the spatial distribution, extent, and explained variance in the different reanalyses are equivalent, suggesting its reliability. The explained variance of this mode for 1979-1998 is much larger than that for 1999-2015; i.e., $\sim 76 \%$ versus $\sim 47 \%$ (the averages of the three reanalyses). This is because the response of HEA to SEA during period 1999-2015 is smaller than in 1979-1998, as will be seen below. In contrast, the EOF1 of HES is equatorially symmetric, with the combined ascending branch at the equator, and two mirrored cells, one in each hemisphere (Fig. 2). The explained variance of the EOF1 of HES in the period 1999-2015 is greater than in the period 19791998 ; i.e., $\sim 56 \%$ versus $\sim 43 \%$. As reported the long-term trend (Wu and Xie 2003) and intensity (Waliser et al. 1999) of the HC in NCEP1 are stronger, it is seen the intensity of the EOF1 in both the NCEP1 and NCEP2 is greater than in the ERAI for both HEA and HES. However, we focus on the response of HC to SST rather than the intensity, and the result is compared between different periods rather than among datasets, the discrepancies in the
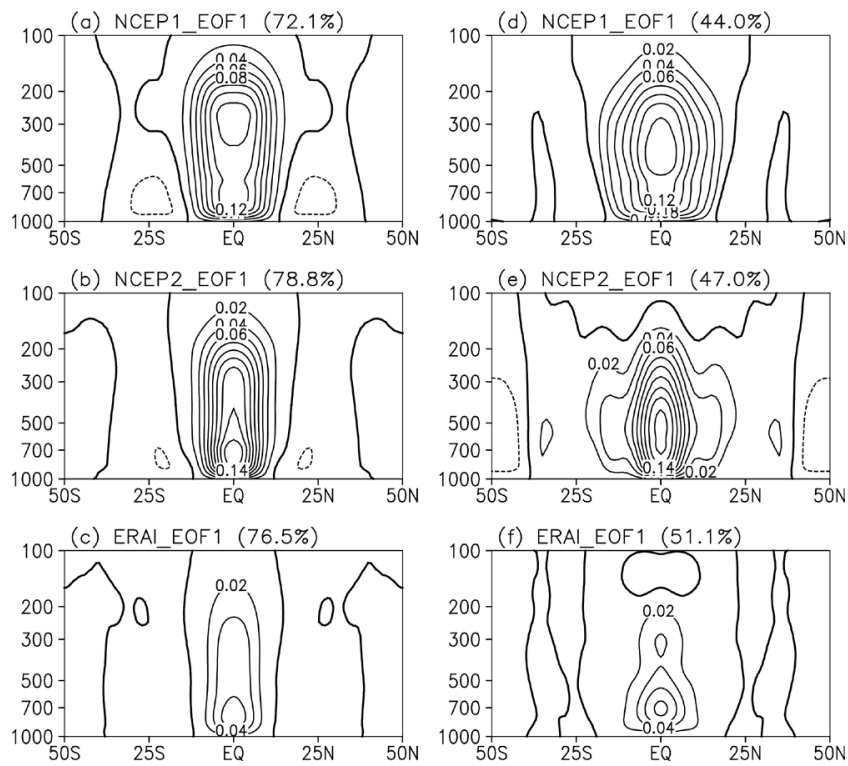

Fig. 1. The principal EOF mode of the equatorially asymmetric component of the Hadley circulation (HEA) during the two periods (left panel) 1979-1998 and (right panel) 1999-2015, with the corresponding explained variances based on the NCEP1 (upper), NCEP2 (middle), and ERAI (below) reanalyses. The contour interval is $0.02 \times 10^{10} \mathrm{~kg} \mathrm{~s}^{-1}$; positive (negative) contours are shown as solid (dotted) lines, and the zero contours are thickened. Clockwise circulation (the northern cell) is defined as positive and anti-clockwise circulation (the southern cell) is defined as negative.
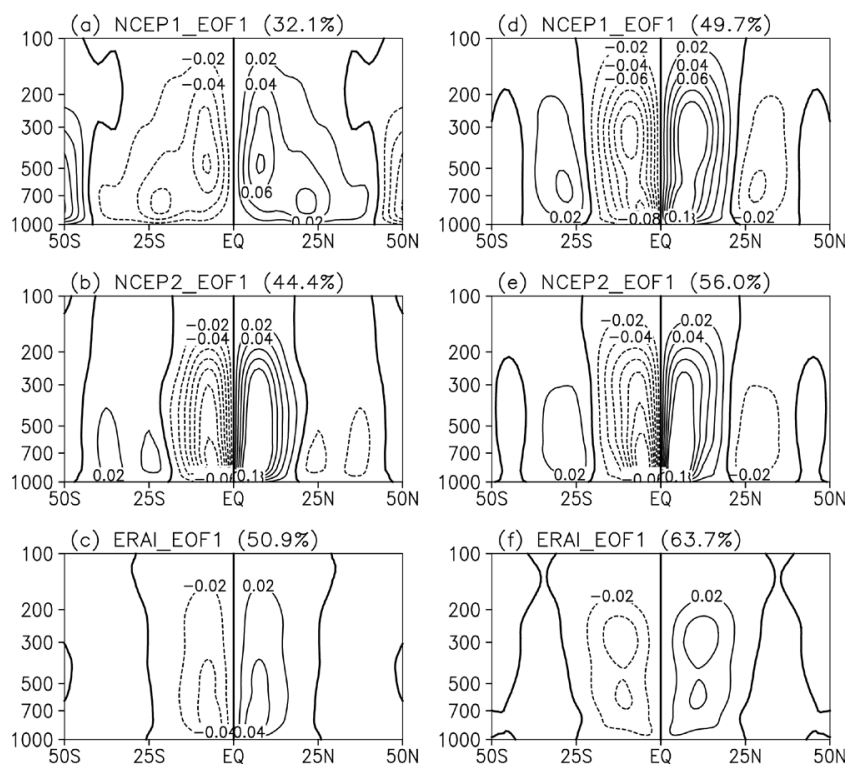

Fig. 2. As in Fig. 1, but for the principal EOF mode of the equatorially symmetric component of the Hadley circulation (HES).

intensity of $\mathrm{HC}$ among different reanalyses are not discussed. In addition, the regression chart of the MSF onto the HEA's (HES's) principal component (PC1) displays an equatorially asymmetric (symmetric) meridional circulation (Fig. S1), which in turn indicates that the decomposition method is reasonable.

The EOF1 of SEA is equatorially asymmetric, with the maximum and minimum located around $10^{\circ} \mathrm{S}$ and $10^{\circ} \mathrm{N}$ during $1979-$ 1998 (Fig. 3a), respectively. However, the maximum and minimum of the EOF1 of SEA during 1999-2015 are at $\sim 20^{\circ} \mathrm{S}$ and $\sim 20^{\circ} \mathrm{N}$ (Fig. 3b), respectively. Since the ascending and descending 

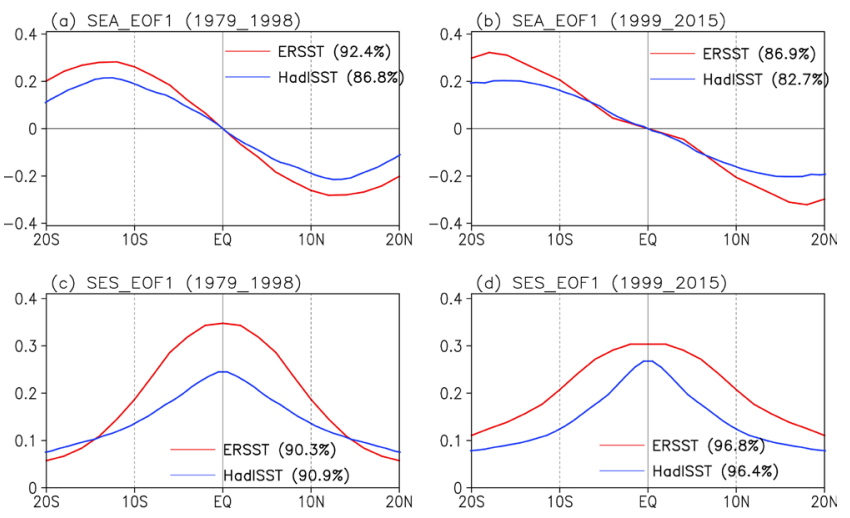

Fig. 3. (a) The principal EOF mode of the equatorially asymmetric component of tropical zonal mean SST for the period 1979-1998. (b) As in Fig. 3a, but for the period 1999-2015. (c)-(d) As in Figs. 3a and 3b but for the equatorially symmetric component. The red and blue lines indicate based on the ERSST and HadISST data, respectively.
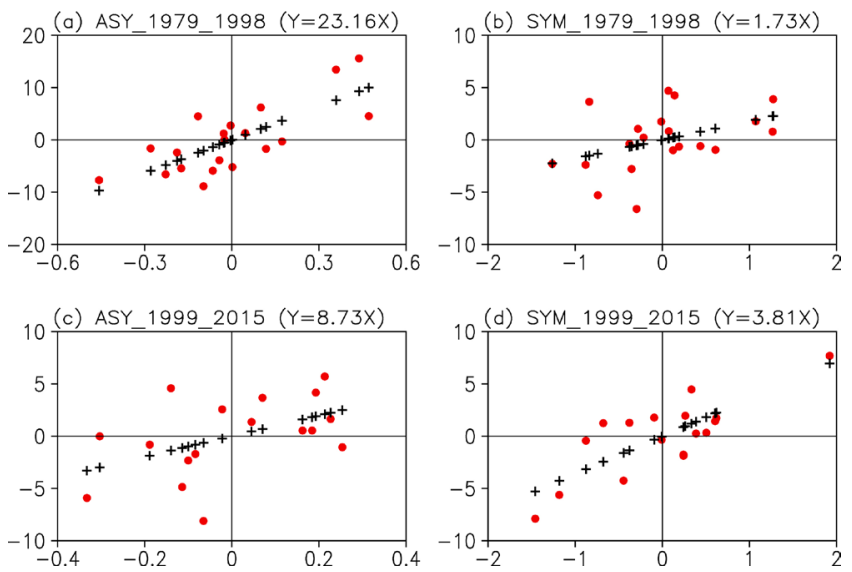

Fig. 4. (a) Scatter plot of the PC1 of the SEA against the PC1 of the HEA, using the ERSST. The red dots are the PCs of HEA and SEA, the black dots are the linear fit of the scatter, for which the regression coefficient is shown. (b) As in Fig. 4a, but for the scatter plot of the PC1 of the SES against the PC1 of the HES. (c)-(d) As in Figs. 4a and 4b, but for the period 1999-2015.

branches of the anomalous $\mathrm{HC}$ are generally aligned with the locations of maximum and minimum meridional SST gradient (Feng et al. 2013; Feng and Li 2013), these results imply that the extent of the HC associated with SEA should be broader during 1999-2015 than 1979-1998, which is consistent with the distribution of the EOF1 as shown in Fig. 1. This point is further verified by the significant correlations of the PCs between HEA and SEA across different reanalyses (with correlation coefficient beyond 0.7 across different reanalyses), and consistent with previous study that the position of the ascending branch of $\mathrm{HC}$ is associated with an anomalous equatorial variation in Inter-tropical convergence zone (e.g. Bischoff and Schneider 2014; Scheinder et al. 2014). The EOF1 of SES is parabolic with the maximum at the equator, decreasing with latitude in each hemisphere in the two periods (Fig. 3c and 3d). The explained variance of this mode is evidently enhanced in 1999-2015 compared with 1979-1998, consistent with the larger explained variance of HES in 1999-2015. In contrast, the explained variances of SEA vary little in the two periods. This is not consistent with the generally decreased explained variances for the EOF1 of HEA as shown in Fig. 1, implying that the response of HEA to SEA may differ in the two periods.

The response of HEA to SEA, and HES to SES, as well as their response ratio in the two periods is shown in Fig. 4. During 1979-1998, a given amplitude variation in SEA is accompanied
Table 1. Regression coefficients of the HEA's (HES's) PC1 with respect to SEA's (SES's) PC1 and their ratio calculated using the various reanalysis datasets within the two periods of 1979-1998 and 1999-2015, respectivey.

\begin{tabular}{|c|c|c|c|c|c|c|}
\hline \multirow{2}{*}{ Data sources } & \multicolumn{3}{|c|}{ ERSST3 (1979-1998) } & \multicolumn{3}{|c|}{ ERSST3 (1999-2015) } \\
\hline & ASY & SYM & ratio & ASY & SYM & ratio \\
\hline NCEP1 & 23.16 & 1.73 & 13.4 & 8.73 & 3.81 & 2.3 \\
\hline NCEP2 & 32.78 & 1.65 & 19.9 & 10.39 & 4.76 & 2.2 \\
\hline ERAI & 80.46 & 10.03 & 8.0 & 41.64 & 12.85 & 3.2 \\
\hline \multirow{2}{*}{ Data sources } & \multicolumn{3}{|c|}{ HadISST (1979-1998) } & \multicolumn{3}{|c|}{ HadISST (1999-2015) } \\
\hline & ASY & SYM & ratio & ASY & SYM & ratio \\
\hline NCEP1 & 14.15 & 1.08 & 13.1 & 7.56 & 2.66 & 2.8 \\
\hline NCEP2 & 19.67 & 1.04 & 18.9 & 6.45 & 3.28 & 2.0 \\
\hline ERAI & 48.11 & 5.82 & 8.3 & 26.97 & 9.22 & 2.9 \\
\hline
\end{tabular}
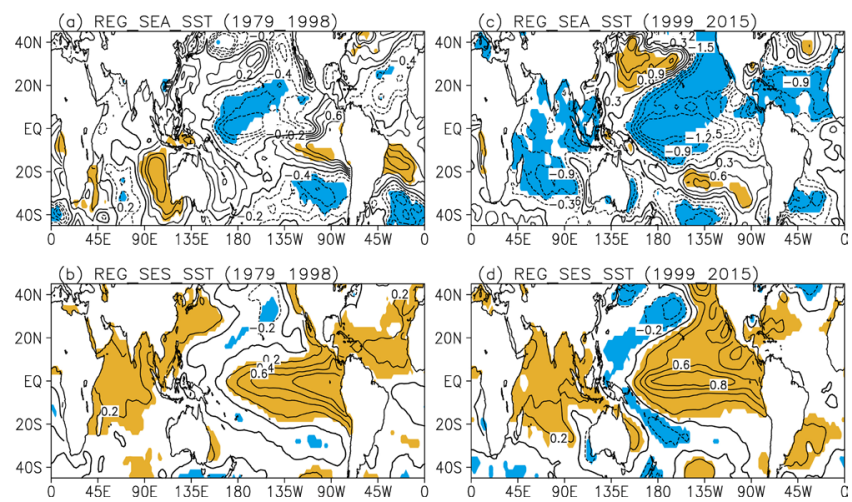

Fig. 5. (a) Regression pattern of SST with respect to the PC1 of the SEA for the period 1979-1998. (b) As in Fig. 5a, but for the regression against the PC1 of the SES. (c)-(d) As in Figs. 5a and 5b, but for the period 19992015. Shading indicates significant at 0.1 level.

by a 23 -unit variation in HEA, but a $~ 2$-unit variation in HES (based on NCEP1). This result indicates that, during 1979-1998, the response of HEA to SEA is about $\sim 10$ times larger than that of HES to SES even when the magnitude of the SST variation is the same. However, for the period 1999-2015, the ratio of the responses between HEA and HES to SST is around $\sim 3$. Similar results are obtained for the magnitude of the response ratio using different reanalyses (Table 1). The differences between the ratios of the response are due to two factors: 1) the enhanced response of HES to SES in the period 1999-2015; and 2) the suppressed response of HEA to SEA during 1999-2015 as consistently seen in Fig. 1. The suppressed response of HEA to SEA during 19992015 provides an explanation for the decreased explained variance of the EOF1 of HEA's year-to-year variability during 1999-2015. Meanwhile, the enhanced explained variance of the EOF1 for HES is due to the strengthened response of HES to SES. This result implies that air-sea interactions within the tropics during the hiatus may change correspondingly, and the combined effects of the responses of HEA and HES to SST lead to a decreased response ratio of the $\mathrm{HC}$ to different meridional structures of SST.

Furthermore, the regional characteristics of tropical SST associated with the SEA and SES are examined to for the different ocean basins (Fig. 5). It is seen that the variation of SES relates to a classical El Niño like pattern during period 1979-1998. By contrast, the maximal correlation center has shifted to the central Pacific during period 1999-2015, which is consistent with the result that the central Pacific El Niño (Yu and Kao 2007) is more frequent in recent decades. As to the SEA, there are significant negative correlations over the central Pacific but centered to the north of the equator during period 1979-1998. By contrast, the 
SEA is linked with negative correlations over central and eastern Pacific, resembling to the La Niña like cooling pattern (Kosaka and Xie 2013; Li et al. 2015). On the other hand, it is known that ENSO plays an important role in regulating the variations of $\mathrm{HC}$ (e.g., Lu et al. 2008; Sun and Zhou 2014). It is reported that both El Niño and La Niña are corresponding to the equatorially symmetric structures (Zhang et al. 2009). Thus, it is possible that the equatorially symmetric variation of SST (i.e., SES) would be largely impacted during the ENSO events, which in turn may play a role in determining the meridional distribution of SST, and alter the response ratio between the equatorially asymmetric and symmetric variations of HC to SST. The result here implies the variation of SST over Pacific may play an important role in determining the reduced response ratio of $\mathrm{HC}$ to SST in the recent hiatus.

In addition, considering the close relationship between the SES and ENSO, and the demarcation year 1998 is right correspond to a noticeable El Niño event, the response contrast of $\mathrm{HC}$ to SST is examined in period 1979-1997. Despite that the response ratio in the two periods (1979-1997 versus 1999-2015) still shows a reduction (e.g. $\sim 9$ versus $\sim 2$ for the NCEP2), it is seen that the response ratio (HEA to SEA versus HES to SES) of including (i.e. 1979-1998) and excluding (i.e. 1979-1997) the year 1998 exhibits an evident decrease (e.g., $\sim 19$ versus $\sim 9$ for the NCEP2). This point highlights the important role of ENSO on the HC ( $\mathrm{Lu}$ et al. 2008), and implies the occurrence of ENSO events plays an essential role in influencing the meridional structure of tropical SST.

\section{Conclusions and discussion}

This paper demonstrates that the contrast in the response of HEA to SEA against HES to SES evidently decreased during the recent hiatus compared with the previous period. The response ratios between HEA to SEA against HES to SES varied from $~ 10$ to $\sim 3$ from the period 1979-1998 to 1999-2015. This decrease in the response contrast by about a factor of three provides a possible explanation for why the explained variance of the HC's principal mode varies greatly in the two periods. Moreover, month-tomonth datasets are further used to examine the reliability of the result after removing the annual cycle. It is found that the result based on month-to-month is consistent with that of the annual mean. The consistent result between the month-to-month and annual mean suggests the length of sample in the manuscript has little impacts on the result. The result implies that the airsea interactions prior to and post 1998 may have changed, as the response of the HC to SST involves strong feedback mechanisms as well as cumulus parameterization processes (Numaguti 1994). Similar changes in air-sea processes during this period have been reported; for instance, changed SST variations over the equatorial Pacific (Kosaka and Xie 2013), and changed ocean interior processes (Chen et al. 2015). In addition, the response contrasts of HC to SST during four seasons are further examined. Despite the response ratios show certain differences among seasons, consistent reduced response ratios between periods 1979-1998 and 1999-2015 are observed. Besides, this study focuses on the zonal mean view, as reported that $\mathrm{HC}$ changes vary considerably regionally, it is worthwhile to further detect the regional variations associated with the HC. Especially, as it seen that the variations of SEA and SES is closely linked with SSTs over Pacific, it is of interest to further establish the role of ENSO on the response of $\mathrm{HC}$ to different SST meridional structures.

Although the decomposition used in the present study is linear, and the equatorially asymmetric and symmetric components are not orthogonal, this method provides a feasible tool to assess the influence of SST on the HC. Since the variation of HEA mainly reflects interdecadal variation, while the HES presents a long term linear trend (Feng et al. 2016), the results indicate that the variation of HEA has been more complex during 1999-2015, and has been modulated by strong interdecadal signals. This is consistent with previous studies that the hiatus is an interdecadal phenomenon (e.g. Li et al. 2013; Kosaka and Xie 2013). How- ever, which of the interdecadal signals influences the HC, in particular, the HEA, is still unknown. Further, the recent hiatus is neither special nor unprecedented; however, there is no adequate atmosphere dataset to explore the earlier hiatus. It is of interest to further explore this point by employing the CMIP5 simulations in the future to detect the characteristics of the response of $\mathrm{HC}$ to different SST meridional structures, compare the similarities and dissimilarities between the recent hiatus and earlier ones, and identify the possible anomalies and physical processes involved.

\section{Acknowledgement}

This work was supported by the Ministry of Science and Technology National Key Research and development Projects of China (2016YFA0601801), SOA Program on Global Change and Air-Sea interactions (GASI-IPOVAI-03), and National Natural Science Foundation of China (41475076). The NCEP/NCAR, NCEP-DOE, ERA-Interim reanalysis and ERSST datasets were obtained from NOAA and are available at http://www.esrl.noaa. gov/psd/data/gridded/. The HadISST dataset was obtained from the UK Met Office Hadley Centre and is available online at http:// www.metoffice.gov.uk/hadobs/hadisst/data/download.html.

Edited by: Y. Kosaka

\section{References}

Adam, O., T. Schneider, and N. Harnik, 2014: Role of changes in mean temperatures versus temperature gradients in the recent widening of the Haley circulation. J. Climate, 27, $7450-7461$.

Bischoff, T., and T. Schneider, 2014: Energetic constraints on the position of the Intertropical Convergence Zone. J. Climate, 27, 4937-4951.

Booth, B. B., and co-authors, 2012: Aerosols implicated as a prime driver of twentieth-century North Atlantic climate variability. Nature, 484, 228-232.

Chen, L. J., F. Zheng, and T. Zhu, 2015: Distinctive ocean interior changes during the recent warming slowdown. Sci. Rep., 5, 14346.

Dai, A., J. C. Fyfe, S. P. Xie, and X. Dai, 2015: Decadal modulation of global-mean temperature by internal climate variability. Nature Clim. Change, 5, 555-559.

Dee, D. P., and co-authors, 2011: The ERA-Interim reanalysis: Configuration and performance of the data assimilation system. Quart. J. Roy. Meteor. Soc., 137, 553-597.

Dima, I. M., and J. M. Wallace, 2003: On the seasonality of the Hadley cell. J. Atmos. Sci., 60, 1522-1526.

England, M. H., and co-authors, 2014: Recent intensification of wind-driven circulation in the Pacific and the ongoing warming hiatus. Nature Clim. Change, 4, 222-227.

Feng, J., and J. P. Li, 2013: Contrasting impacts of two types of ENSO on the boreal spring Hadley circulation. J. Climate, 26, 4773-4789.

Feng, J., J. P. Li, and F. Xie, 2013: Long-term variation of the principal mode of boreal spring Hadley circulation linked to SST over the Indo-Pacific warm pool. J. Climate, 26, 532544.

Feng, J., and co-authors, 2016: Contrasting responses of the Hadley circulation to equatorially asymmetric and symmetric meridional sea surface temperature structures. $J$. Climate, 29, 8949-8963.

Feng, J., and co-authors, 2017: The responses of the Hadley circulation to different meridional SST structures in the seasonal cycle. J. Geophys. Res. Atmos., 122, 7785-7789, doi: 10.1002/2017JD026953.

Feng, R., J. P. Li, and J. C. Wang, 2011: Regime change of the boreal summer Hadley circulation and its connection with the tropical SST. J. Climate, 24, 3867-3877.

Guo, Y. P., 2016: The interdecadal variation of the Hadley circulation and its relationship with the meridional gradient of sea 
surface temperature. D.C. thesis, NanJin University, $190 \mathrm{pp}$.

Guo, Y. P., and co-authors, 2016: The multidecadal variability of the asymmetric mode of the boreal autumn Hadley circulation and its link to the Atlantic Multidecadal Oscillation. $J$. Climate, 29, 5625-5641.

Hou, A. Y., and R. S. Lindzen, 1992: The influence of concentrated heating on the Hadley circulation. J. Atmos. Sci., 49, 12331241.

Kalnay, E., and co-authors, 1996: The NCEP/NCAR 40-Year Reanalysis Project. Bull. Amer. Meteor. Soc., 77, 437-472.

Kanamitsu, M., and co-authors, 2002: NCEP-DOE AMIP-II Reanalysis (R-2). Bull. Amer. Meteor. Soc., 83, 1631-1643.

Keenlyside, N. S., and co-authors, 2008: Advancing decadal-scale climate prediction in the North Atlantic sector. Nature, 453, 84-88.

Kosaka, Y., and S. P. Xie, 2013: Recent global-warming hiatus tied to equatorial Pacific surface cooling. Nature, 501, 403407.

Li, J. P., C. Sun, and F. F. Jin, 2013: NAO implicated as a predictor of Northern Hemisphere mean temperature multidecadal variability. Geophys. Res. Lett., 40, 5497-5502.

Li, Y., and co-authors, 2015: Ocean dynamical processes associated with the tropical Pacific cold tongue mode. J. Geophys. Res. Oceans, 120, 6149-6435, doi:10.1002/2015JC010814.

Lu, J., G. Chen, and D. M. Frierson, 2008: Response of the zonal mean atmospheric circulation to El Niño versus Global Warming. J. Climate, 21, 5835-5851.

Mitas, C. M., and A. Clement, 2006: Recent behavior of the Hadley cell and tropical thermodynamics in climate models and reanalyses. Geophys. Res. Lett., 33, L01810.

Numaguti, A., 1994: Dynamics and energy balance of the Hadley circulation and the tropical precipitaton zones, Part II: sensitivity to meridional SST distribution. J. Atmos. Sci., 52, $1128-1141$.

Rayner, N. A., and co-authors, 2003: Global analyses of sea surface temperature, sea ice, and night marine air temperature since the late nineteenth century. J. Geophys. Res., 108, D14 4407.

Schneider, E., and R. S. Lindzen, 1977: Axially symmetric steady state models of the basic state of instability and climate studies, Part I: Linearized calculations. J. Atmos. Sci., 34, 253-279.

Schneider, T., T. Bischoff, and G. H. Haug, 2014: Migrations and dynamics of the Intertropical Convergence Zone. Nature, $\mathbf{5 1 3}, 45-53$

Smith, T. M., and co-authors, 2008: Improvements to NOAA's historical merged land-ocean surface temperature analysis (1880-2006). J. Climate, 21, 2283-2296.

Sun, Y., and T. J. Zhou, 2014: How does El Niño affect the interannual variability of the boreal summer Hadley Circulation? J. Climate, 27, 2622-2642.

Trenberth, K. E., and J. T. Fasullo, 2013: An apparent hiatus in global warming? Earth's Future, 1, 19-32.

Waliser, D. E., and co-authors, 1999: The Hadley circulation: Assessing NCEP/NCAR reanalysis and sparse in-situ estimates. Climate Dyn., 15, 719-735.

Wu, R., and S. P. Xie, 2003: On equatorial Pacific surface wind changes around 1977: NCEP-NCAR reanalysis versus COADS observations. J. Climate, 16, 167-173.

Xing, N., J. P. Li, and L. N. Wang, 2016: Multidecadal trends in large-scale annual mean SATa based on CMIP5 historical simulations and future projections. Engineering, 3, 136143, doi:10.1016/J.ENG.2016.04.011.

Yu, J. Y., and H. Y. Kao, 2007: Decadal changes of ENSO persistence barrier in SST and ocean heat content indices: 1958-2001. J. Geophys. Res., 112, D13106.

Zhang, W. J., J. P. Li, and F. F. Jin, 2009: Spatial and temporal features of ENSO meridional scales. Geophys. Res. Lett., 36, L15605, doi:10.1029/2009GL038672.

Manuscript received 11 July 2017, accepted 11 September 2017

SOLA: https://www.jstage.jst.go.jp/browse/solal 